\title{
Sintomas neuropsicológicos e biomarcadores liquóricos na Demência da doença de Alzheimer leve e Comprometimento Cognitivo Leve amnéstico
}

\author{
Katienne dos Santos Antonio*, Larissa dos Santos Fonseca*, Thamires N. C. Magalhães, Camila V. L. Teixeira \\ Marcio L. F. Balthazar.
}

\begin{abstract}
Resumo
A doença de Alzheimer é caracterizada por problemas cognitivos que limitam a independência do indivíduo. No comprometimento cognitivo leve amnéstico há problemas de memória episódica que ainda não limitam as atividades funcionais. Nesse estudo, avaliamos se os principais biomarcadores da DA no líquor (proteínas beta amiloite, tau total e p-tau) influenciam o desempenho cognitivo no teste Miniexame do Estado Mental (MEEM). Encontramos que apenas a proteína tau total prediz o desempenho no MEEM, assim como a idade e escolaridade.
\end{abstract}

\section{Palavras-chave \\ Biomarcadores, Alzheimer,CCL.}

\section{Introdução}

A Doença de Alzheimer (DA) é a causa mais frequente de demência (cerca de 50 a $70 \%$ dos casos), afetando a cognição e comprometendo a independência do indivíduo. ${ }^{1}$

Sendo considerada uma doença multifatorial, ela altera principalmente a memória, podendo comprometer a fala, raciocínio, socialização, visão, escrita, orientação temporal e habilidades visuoespaciais. ${ }^{2}$

Comprometimento cognitivo leve amnéstico (CCLa) é uma condição clínica em que há problemas objetivos em memória episódica mas que não comprometem significativamente a independência do indivíduo. Pacientes com CCLa têm risco aumentado de evoluir para demência da DA.

Dentre os principais agentes fisiopatológicos da DA estão as placas neuríticas, cujo núcleo é constituído de proteína beta-amiloide (BA), e os emaranhados neurofibrilares, constituídos por proteína tau hiperfosforilada. Embora esse processo seja bem conhecido, não é clara como a alteração dessas proteínas afeta os processos cognitivos na DA e no CCLa. ${ }^{2}$

Assim, nesse estudo, objetivamos avaliar se: 1) há diferença nos níveis das proteínas BA, tau fosforilada (p-tau) e tau total (t-tau) no líquor de pacientes com demência da DA em fase leve e CCLa; 2) se há correlações significativas entre o desempenho em teste cognitivo e os níveis dessas proteínas liquóricas nos 2 grupos.

\section{Resultados e Discussão}

Foram incluídos 123 pacientes com CCLa e 97 pacientes com demência da DA em fase leve. Todos os pacientes foram submetidos a punção liquórica para dosagem das proteínas BA, p-tau e t-tau e avaliação neuropsicológica completa, com testes de memória, linguagem, orientação e funções executivas. Nesse estudo, realizamos teste de regressão linear múltipla considerando como variável dependente uma medida de cognição geral, o Miniexame do Estado Mental (MEEM) e como variáveis independentes, as dosagens de BA, p-tau e t-tau, além de idade e anos de escolaridade.
Como resultados, verificamos que os pacientes com DA tiveram desempenho pior no MEEM que os pacientes com CCLa, eram mais velhos e menos escolarizados. Em relação aos níveis das proteínas liquóricas, encontramos diferenças apenas nos níveis de ttau (pacientes com DA com mais t-tau que CCLa).

Como resultado da regressão múltipla, verificamos que as variáveis idade, educação e t-tau predisseram o desempenho no MEEM. Não houve resultado significativamente positivo em relação às proteínas BA e p-tau.

Conforme esperado, os pacientes com DA tiveram desempenho inferior aos pacientes com CCLa no MEEM. Porém, eram também mais velhos e menos escolarizados, o que pode ter influenciado no desempenho do teste. Não encontramos diferenças nos níveis de BA e p-tau entre os grupos. Esse achado mostra que nossos pacientes com CCLa realmente apresentam a fisiopatologia da DA, podendo ser caracterizados como CCLa devido à doença de Alzheimer. Em relação à t-tau, nossos pacientes com DA apresentaram maiores níveis no líquido cefalorraquidiano (LCR), o que indica que estão em uma fase mais avançada de doença cerebral.

Ainda, encontramos que o aumento de t-tau, indicando maior lesão cerebral, prediz o desempenho no MEEM, assim como a idade e educação. Como limitação do estudo, há diferença na idade entre os grupos que pretendemos corrigir. Também, planejamos ampliar 0 número de testes para verificar possíveis correlações com as proteínas liquóricas.

\section{Conclusões}

Nosso estudo mostrou que há diferença no desempenho do MEEM e nas medidas de t-tau entre os 2 grupos. Idade, escolaridade e t-tau predisseram o desempenho no MEEM.

1 ORTIZ, K. Z. Distúrbios Neurológicos Adquiridos. Barueri, SP: Manole, 2010 2ed. 308p. 484p.

2 FORLENZA, O. V; CARAMELLI, P. Neuropsiquiatria Geriátrica. São Paulo: Editora Atheneu, 2000. 24p. 695p. 